\title{
ENDOSCOPY
}

\section{Endoscopic transpapillary bile duct biopsy with the combination of intraductal ultrasonography in the diagnosis of biliary strictures}

\author{
K Tamada, T Tomiyama, S Wada, A Ohashi, Y Satoh, K Ido, K Sugano
}

See end of article for authors' affiliations

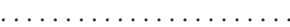

Correspondence to: Dr K Tamada, Department of Gastroenterology, Jichi Medical School, Yakushiii, Tochigi 329-0498, Japan

Accepted for publication 15 May 2001

\begin{abstract}
Background: When endoscopic retrograde cholangiopancreatography (ERCP) guided bile duct biopsy fails to demonstrate malignancy, it remains unclear how to manage patients with presumably malignant strictures.

Aims: To evaluate the value of intraductal ultrasonography (IDUS) when bile duct biopsy is negative. Methods: Sixty two patients with strictures of the bile duct were studied prospectively. During ERCP, IDUS was performed using an ultrasonic probe (diameter $2.0 \mathrm{~mm}$; frequency $20 \mathrm{MHz}$ ). Following IDUS, a bile duct biopsy was performed using forceps (diameter $1.8 \mathrm{~mm}$ ). The IDUS images of the tumour were classified as polypoid lesions, localised wall thickening, intraductal sessile tumours, sessile tumour outside of the bile duct, or absence of apparent lesion. The bile duct wall structures at the site of the tumour as well as the maximum diameter of the tumour were also analysed. The IDUS findings were compared with the histological findings or clinical course.

Results: When the IDUS images showed a polypoid lesion $(n=19)$, localised wall thickening $(n=8)$, intraductal sessile tumour $(n=13)$, and sessile tumour outside of the bile duct $(n=20)$, the sensitivities of the biopsy were $80 \%, 50 \%, 92 \%$, and $53 \%$, respectively. Multiple regression analysis showed that the presence of sessile tumour (intraductal or outside of the bile duct: $p<0.05$ ), tumour size greater than $10.0 \mathrm{~mm}(p<0.001)$, and interrupted wall structure $(p<0.05)$ were independent variables that predicted malignancy.

Conclusion: When biopsy fails to demonstrate evidence of malignancy, the presence of sessile tumour (intraductal or outside of the bile duct), tumour size greater than $10.0 \mathrm{~mm}$, and interrupted wall structure on IDUS images are factors that can predict malignancy.
\end{abstract}

stis issue sampling is essential for the treatment of biliary strictures. ${ }^{1-12}$ Transpapillary bile duct biopsy during endoscopic retrograde cholangiopancreatography (ERCP) is a simple, safe, and effective way to biopsy these strictures. ${ }^{1-7}$ However, when biopsy fails to demonstrate malignancy, there are no clear guidelines for further management of these patients. In this study, we evaluated the role of intraductal ultrasonography (IDUS) $)^{13-36}$ in an effort to clarify these strictures when ERCP biopsy is negative. IDUS, using a high frequency thin calibre ultrasonic probe inserted into the bile duct, produces a high quality cross sectional image that is useful for further characterisation of biliary tract cancers. ${ }^{13-36}$

\section{PATIENTS AND METHODS}

\section{Patients}

Between December 1995 and February 2001, 62 patients with extrahepatic bile duct lesions detected by ERCP underwent transpapillary IDUS with bile duct biopsy and were followed prospectively. Patients with lesions of the papilla were excluded. They were 46 men and 16 women, and mean age was 63 (range 33-87) years. Thirty nine patients underwent ERCP for further evaluation of jaundice. The other 19 patients underwent ERCP for evaluation of abdominal discomfort following an abnormal abdominal ultrasonography, showing an intraductal mass $(n=1)$, gall bladder mass $(n=2)$, gall bladder stone $(n=1)$, and bile duct dilatation $(n=15)$. The remaining four patients were found to have an intraductal mass $(n=3)$ or intrahepatic bile duct dilatation $(\mathrm{n}=1)$ during ultrasonography on routine examination. The final diagnoses included the following: bile duct cancer $(n=25)$, gall bladder cancer $(n=6)$, carcinoma of the pancreatic head $(n=10)$, hepatocellular carcinoma $(n=2)$, gastric cancer $(n=1)$, benign bile duct stenosis secondary to common bile duct stones $(n=7)$, polypoid lesion of the distal bile duct $(n=5)$, inflammatory polyp of the common bile duct $(\mathrm{n}=1)$, benign biliary stenosis secondary to a traffic accident injury $(n=1)$, primary sclerosing cholangitis $(\mathrm{n}=1)$, Mirizzi's syndrome $(\mathrm{n}=1)$, and chronic pancreatitis $(\mathrm{n}=2)$.

In 44 of the 62 patients with malignant biliary lesions, the diagnosis of malignancy was confirmed by surgical resection $(n=29)$, aspiration biopsy $(n=4)$, endoscopic duodenal biopsy $(n=1)$, endoscopic gastric biopsy $(n=1)$, percutaneous transluminal biopsy $(n=1)$, endoscopic biliary biopsy $(n=7)$, and bile cytology $(n=1)$. Of the 18 patients with benign bile duct lesions, two underwent surgical resection. The remaining 16 patients were clinically observed for at least 18 months.

\section{Methods}

Written informed consent was obtained from all patients before ERCP, IDUS, and biopsy. Approval for the study from our institutional review board was not required as biopsy and IDUS are considered standard care for these patients.

ERCP was performed using a conventional duodenoscope (JF-200 or JF-230; Olympus Optical Co., Tokyo, Japan). The stenotic area was cannulated using a 0.025 inch diameter polymer coated guidewire (Radifocus; Terumo Co., Tokyo, Japan). After exchanging the polymer coated guidewire for a

Abbreviations: ERCP, endoscopic retrograde cholangiopancreatography; IDUS, intraductal ultrasonography; ENBD, endoscopic nasobiliary drainage; HCC, hepatocelular carcinoma; PTCS, percutaneous transhepatic cholangioscopy. 


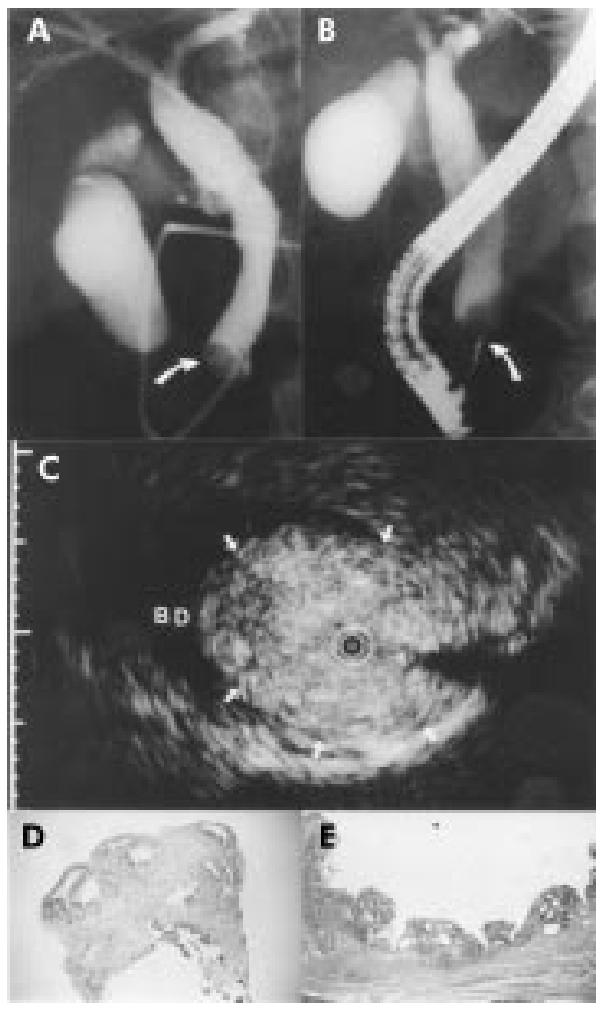

Figure 1 A 60 year old man with a polypoid lesion of the bile duct. The patient initially presented with obstructive jaundice and underwent endoscopic retrograde cholangiography. (A)

Cholangiography showed a filling defect in the distal portion of the bile duct (arrow). (B) Endoscopic transpapillary bile duct biopsy was performed (arrow). (C) A frame from intraductal ultrasonography showed the probe in the bile duct (BD) within the ultrasonographic field of view. Note the narrow based polypoid lesion (arrows) with the normal structure of the bile duct. The narrow dot at the margin of the frame was $1.0 \mathrm{~mm}$ in width. (D) The histological findings of the biopsy specimen showed only inflammation (haematoxylin and eosin, $\times 10$ ). (E) The histological findings of the resected specimen showed inflammatory polyp (haematoxylin and eosin, $\times 2$ ).

stiff Zebra guidewire (Microvasive Co., Watertown, Massachusetts, USA), a $2.0 \mathrm{~mm}$ diameter ultrasonic probe with a frequency of $20 \mathrm{MHz}$ (MP-PN20-06L; Aloka Co., Tokyo, Japan) was inserted into the bile duct along the guidewire.
Endoscopic sphincterotomy was not performed. The tip of the probe had a side slit for the guidewire. The catheter generated high resolution, real time, cross sectional images with an axial resolution of $0.1 \mathrm{~mm}$ and a maximum penetration of approximately $20 \mathrm{~mm}$. The IDUS images were recorded on S-VHS videotapes and individual still frames. After the ultrasonic probe was removed, an endobiliary biopsy was performed under fluoroscopic guidance using a clamshell-type needleless biopsy forceps (FB-39Q; Olympus Optical) with a Teflon sheath (outer diameter $1.8 \mathrm{~mm}$ ). While maintaining the guidewire over the stricture, the biopsy forceps were inserted into the orifice of the papilla along the upper side of the placed guidewire without endoscopic sphincterotomy $(n=35)$. When the forceps could not be smoothly inserted into the orifice of the papilla, an Olbert 5 French balloon tipped biliary catheter (Microvasive) with a length of $180 \mathrm{~cm}$ and a balloon length of $3 \mathrm{~cm}$ (maximum diameter $8 \mathrm{~mm}$ ) was passed over the guidewire and endoscopic papillary balloon dilation (balloon sphincteroplasty) was performed using previously reported techniques. ${ }^{37-39}$ During and after the biopsy, the guidewire remained in that position. Three or more specimens were obtained from each patient. After biopsy, an endoscopic nasobiliary drainage (ENBD) tube was introduced along the guidewire when the bile duct was obstructed. During the initial study period, the biopsy was performed following withdrawal of the guidewire as we mistakenly considered that the guidewire would interfere with insertion of the forceps into the papilla $(n=14)$. In the last cases $(n=13)$, a new ropeway-type $1.8 \mathrm{~mm}$ diameter clamshell-type needleless biopsy forceps (prototype, Olympus) were used. The tip of the ropeway-type forceps had a side slit for a guidewire ${ }^{40}$ and therefore the guidewire was left in place during the biopsy.

Biopsy specimens were fixed in $1 \%$ formalin, embedded in paraffin, and stained with haematoxylin and eosin. When the histological examination of the biopsy specimen showed only atypical cells, the results were judged negative for malignancy.

\section{Study design}

The IDUS images were prospectively reviewed by two experts without information from other imaging tests, except for abdominal ultrasonography and cholangiography. The morphology of the tumour was classified as one of the following: polypoid lesion; localised wall thickening; intraductal sessile tumour; sessile tumour outside of the bile duct; or no apparent lesion. Typical cases are presented in figs $1-4$. The bile duct wall structures at the site of the tumour as well as the maximum diameter of the tumour were analysed also. Analysis was
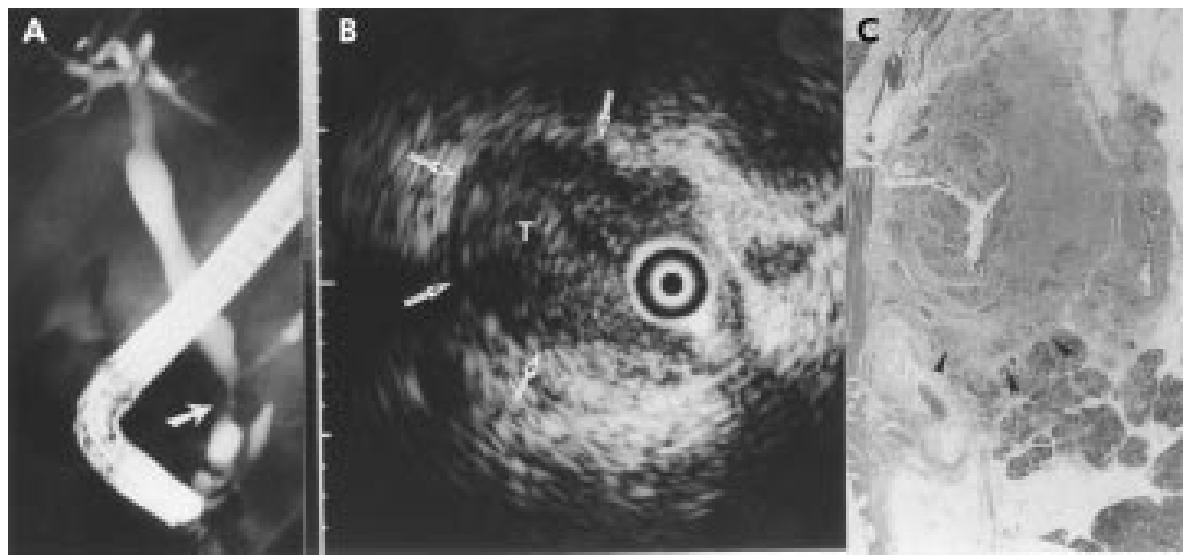

Figure 2 A 66 year old man with a intraductal sessile tumour of the bile duct. The patient initially presented with upper abdominal pain. Ultrasonography showed bile duct dilatation. (A) Cholangiography showed a stenosis at the distal end of the bile duct (arrow). (B) A frame from intraductal ultrasonography showed the probe in the bile duct within the ultrasonographic field of view. Note the intraductal sessile tumour which invaded the pancreatic parenchyma (arrows). The narrow dot at the margin of the frame was $1.0 \mathrm{~mm}$ in width. Endoscopic transpapillary bile duct biopsy showed no evidence of malignancy. (C) The histological findings of the resected specimen showed cholangiocarcinoma which invaded the pancreatic parenchyma (arrowheads) (haematoxylin and eosin, $\times 2$ ). 


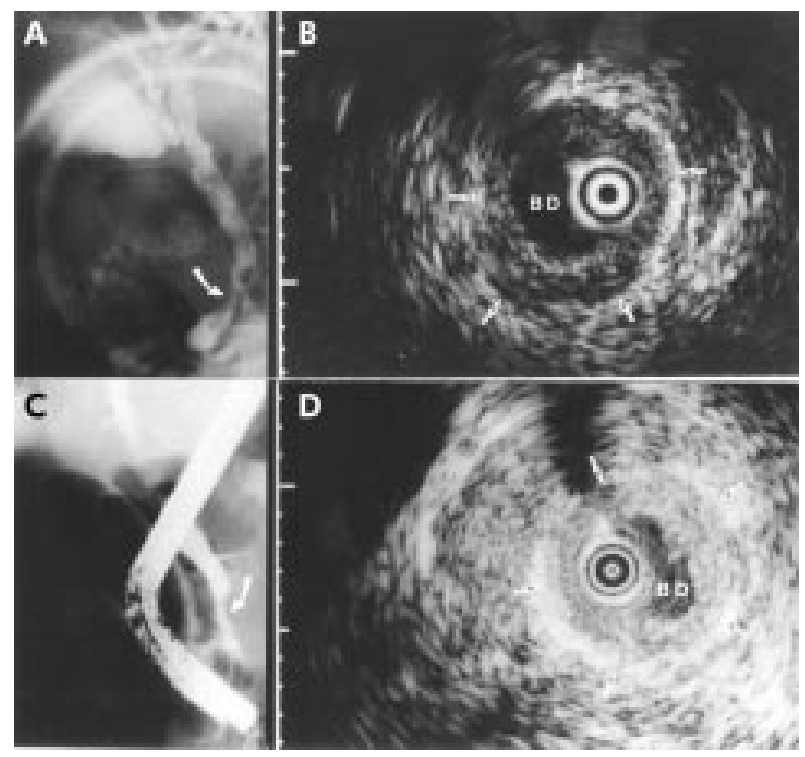

Figure 3 A 57 year old man with localised wall thickening of the bile duct. The patient initially presented with upper abdominal pain. Ultrasonography showed bile duct dilatation. He underwent endoscopic lithotripsy following an endoscopic retrograde cholangiography that showed a bile duct stone. (A)

Cholangiography after endoscopic lithotripsy showed a stenosis in the distal portion of the bile duct (arrow). (B) A frame from the intraductal ultrasonogram showing the probe in the bile duct (BD) within the ultrasonographic field of view. Note the localised bile duct wall thickening (arrows) with the normal structure of the bile duct. The narrow dot at the margin of the frame was $1.0 \mathrm{~mm}$ in width. Endoscopic transpapillary bile duct biopsy showed no evidence of malignancy. (C) Three months later, cholangiography showed persistent stenosis (arrow). (D) A frame from the intraductal ultrasonogram showed wall thickening (arrows). It showed no interval change. Endoscopic transpapillary bile duct biopsy again showed no evidence of malignancy.

performed using the Stat View software package. The data were analysed using Fisher's exact test. Relative significance was analysed by multivariate analysis (multiple regression). A $\mathrm{p}$ value $<0.05$ was considered significant.

\section{RESULTS}

\section{Success rate for insertion of biopsy forceps}

During the initial study period, when the forceps were inserted without the guidewire in place, endoscopic papillary balloon dilation was required in six of 14 patients. During the latter period of the study, when the ropeway-type forceps (prototype) were used, endoscopic papillary balloon dilation was not required in any case. In other patients in whom standard forceps were inserted along the upper side of the placed guidewire, endoscopic papillary balloon dilation was required in three of 35 patients to allow for insertion of the forceps. As a result, the biopsy forceps were successfully inserted into the bile duct after IDUS, and tissue materials were successfully obtained in all patients.

\section{Relationship between IDUS and histology}

The results are summarised in tables $1-3$.

When IDUS showed a polypoid lesion, the biopsy was sensitive in diagnosing malignancy in $80 \%(8 / 10)$ of patients. It was sensitive in all patients who had polypoid lesions with cholangiocarcinoma $(n=8)$. The two patients with false negative biopsy results had tumour thrombi from hepatocellular carcinomas (HCC). In the intrapancreatic bile duct, only two of nine patients with polypoid lesions had malignancy. On the other hand, in the extrapancreatic bile duct, eight of nine patients with polypoid lesions had malignancy.

When IDUS showed a intraductal sessile tumour, all patients had histological evidence of malignancy. All intraductal sessile tumour showed an interruption of wall structures on IDUS findings. Biopsy was sensitive in 92\% (12/13) of patients.

When IDUS showed localised wall thickening, 75\% (6/8) of patients had benign diseases; of the two patients with malignancy, the biopsy was sensitive in only $50 \%(1 / 2)$. Five of six patients with benign strictures that were described as localised wall thickening by IDUS had some clinical origin of their stenosis (two with bile duct stones, one with outmobile injury, one with chronic pancreatitis, and one with Mirizzi's syndrome). On the other hand, two of three patients without evidence of a clinical origin of the stenosis had malignancy.

When IDUS showed sessile tumour outside of the bile duct, $95 \%(19 / 20)$ of patients had malignant diseases, and biopsy was sensitive in 53\% (10/19) of patients. Biopsy was sensitive in $50 \%(6 / 12)$ of patients with pancreatic cancer.

Multiple regression analysis showed that sessile tumour (intraductal or outside of the bile duct: $p<0.05$ ), tumour size $(\mathrm{p}<0.001)$, and interrupted wall structure $(\mathrm{p}<0.05)$ were independent variables that predicted malignancy.

As shown in table 2, when the IDUS finding of interrupted wall structure was used as the criterion of malignancy, the accuracy, sensitivity, and specificity of IDUS were $81 \%, 75 \%$,
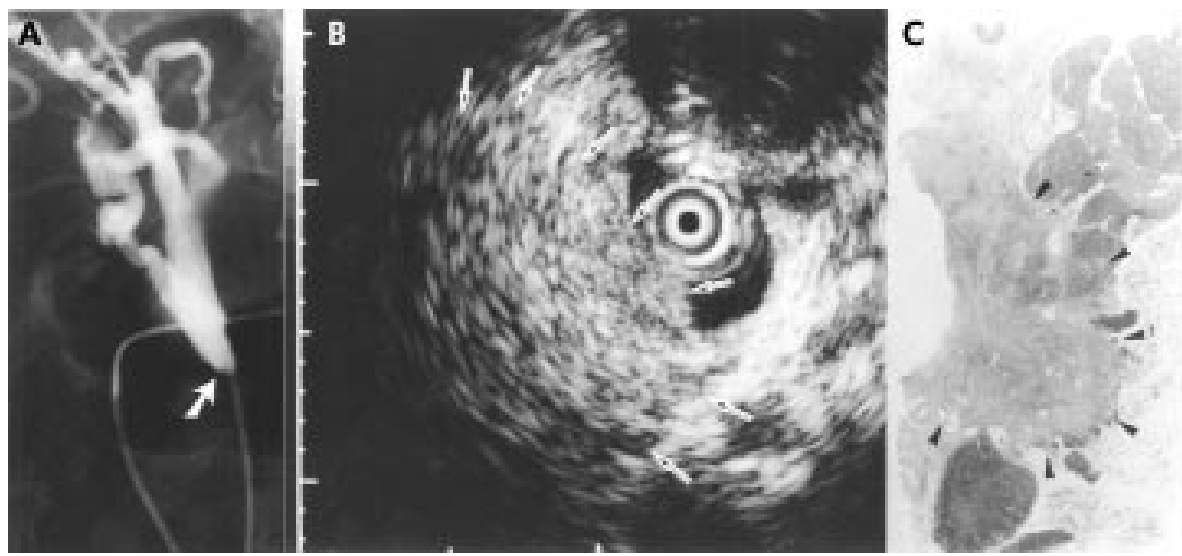

Figure 4 A 64 year old woman with a sessile tumour outside of the bile duct. The patient initially presented with obstructive jaundice and underwent endoscopic retrograde cholangiography. (A) Cholangiography showed a stenosis at the intrapancreatic bile duct (arrow). (B) A frame from intraductal ultrasonography showed the probe in the bile duct within the ultrasonographic field of view. Note the fumour located outside of the bile duct (arrows). The narrow dot at the margin of the frame was $1.0 \mathrm{~mm}$ in width. Endoscopic transpapillary bile duct biopsy showed no evidence of malignancy. (C) The histological findings of the resected specimen revealed pancreatic cancer (arrowheads) that had invaded the bile duct (haematoxylin and eosin, $\times 1$ ). 
Table 1 Morphology on intraductal ultrasonography (IDUS) findings and final diagnosis

\begin{tabular}{|c|c|c|c|c|}
\hline \multirow[b]{3}{*}{ Morphology on IDUS } & \multicolumn{3}{|c|}{ *Final diagnosis } & \multirow[b]{3}{*}{ Total } \\
\hline & \multicolumn{2}{|c|}{ Malignant } & \multirow[b]{2}{*}{ Benign } & \\
\hline & $\begin{array}{l}\text { Biopsy } \\
\text { positive }\end{array}$ & $\begin{array}{l}\text { Biopsy } \\
\text { negative }\end{array}$ & & \\
\hline \multicolumn{5}{|l|}{ Without sessile tumour } \\
\hline No apparent lesion & 0 & 0 & 2 & 2 \\
\hline Localised wall thickening & 1 & 1 & $6^{a}$ & 8 \\
\hline Polypoid lesion & 8 & $2^{b}$ & 9 & 19 \\
\hline \multicolumn{5}{|l|}{ Sessile tumour } \\
\hline Intraductal sessile tumour & 12 & 1 & 0 & 13 \\
\hline Sessile tumour outside of the bile duct & 10 & $9 c$ & $1^{d}$ & 20 \\
\hline Total & 31 & 13 & 18 & 62 \\
\hline
\end{tabular}

'Two with bile duct stone, one with post out mobile injury, one with chronic pancreatitis, one with primary sclerosing cholangitis, and one with Mirizzi's syndrome; btwo with bile duct thrombi by hepatocellular carcinoma; csix with pancreatic cancer, two with peripheral cholangiocarcinoma, and one with gall bladder cancer; and done with tumour forming pancreatitis.

*The process of final diagnosis is shown in table 4.

Table 2 Bile duct wall structure on intraductal ultrasonography (IDUS) findings and final diagnosis

\begin{tabular}{|c|c|c|c|}
\hline \multirow[b]{2}{*}{ Morphology on IDUS } & \multicolumn{2}{|c|}{ *Final diagnosis } & \multirow[b]{2}{*}{ Total } \\
\hline & Malignant & Benign & \\
\hline \multicolumn{4}{|l|}{ Wall structure } \\
\hline Preserved & 11 & 17 & 28 \\
\hline Interrupted & 33 & $1^{a}$ & 34 \\
\hline
\end{tabular}

aOne with tumour forming pancreatitis.

*The process of final diagnosis is shown in table 4.

\begin{tabular}{lccc} 
Table 3 & Maximum tumour size on intraductal \\
ultrasonography (IDUS) findings and final diagnosis \\
\hline \multicolumn{4}{c}{ *Final diagnosis } \\
\cline { 2 - 3 } Maximum tumour size on IDUS & \multicolumn{4}{c}{ Malignant } & Benign & Total \\
\hline Maximum fumour diameter or maximum wall thickening \\
$<10.0 \mathrm{~mm}$ & 7 & 15 & 22 \\
$\geqslant 10.0 \mathrm{~mm}$ & 37 & $3^{\circ}$ & 40 \\
\hline
\end{tabular}

a One with inflammatory polyp, one with polypoid lesion located at the distal end of the bile duct, and one with tumour forming pancreatitis.

*The process of final diagnosis is shown in table 4.

and $94 \%$, respectively. As shown in table 3, when the IDUS finding of a tumour greater than $10 \mathrm{~mm}$ was used as the criterion of malignancy, the accuracy, sensitivity, and specificity of IDUS were $84 \%, 84 \%$, and $83 \%$, respectively.

\section{Course of the patients}

The clinical courses of the patients and the process of final diagnosis are summarised in table 4 . Sixteen patients were judged as having no biliary tract carcinoma and were clinically observed. Two required transient replacement of their biliary endoprosthesis. Seven other patients underwent lithotripsy under endoscopic papillary balloon dilatation. One patient with Mirizzi's syndrome was observed after laparoscopic cholecystectomy. In seven of these 16 patients, a repeat IDUS and biopsy were performed 1-6 months later (mean three months). Improvement in wall thickening or a decrease in the size of the polypoid lesion was seen in five of seven observed patients. The remaining nine of 16 patients rejected a second

\section{Table 4 Course of the patients}

Surgical treatment with diagnosis of malignancy $(n=31)$

Cholangiocarcinoma $(\mathrm{n}=21)$

Polypoid lesion $(n=7)$

Intraductal sessile tumour ( $n=12)$

Localised wall thickening $(n=1)$

Sessile tumour outside of the bile duct ( $n=1)$

Gall bladder cancer $(n=3)$

Sessile tumour outside of the bile duct $(n=3)$

Pancreatic cancer $(n=5)$

Sessile tumour outside of the bile duct $(n=5)$

Inflammatory polyp $(n=1)$

Polypoid lesion ( $n=1)$

Tumour forming pancreatitis $(n=1)$

Sessile tumour outside of the bile duct $(n=1)$

Conservative treatment (stenting) with diagnosis of malignancy $(n=15)$

Evidence of adenocarcinoma

Transpapillary bile duct biopsy $(n=7)$

Percutaneous bile duct biopsy ( $n=1)$

Gastric or duodenal biopsy $(n=2)$

Pancreas aspiration biopsy $(n=2)$

Bile juice cytology $(n=1)$

Evidence of hepatocellular carcinoma

Liver aspiration biopsy $(n=2)$

Follow up by IDUS and biopsy with diagnosis of benign disease $(\mathrm{n}=7)$

Decrease in tumour size on IDUS images $(n=5)$

No change on IDUS images $(n=2)$

Follow up with diagnosis of benign disease, second ERC was rejected $(n=9)$

No symptoms during the period $(n=9)$

ERC, endoscopic retrograde cholangiography.

ERCP as they were asymptomatic. Ultrasonography and laboratory data showed no signs of biliary obstruction in these patients. No signs of malignancy were detected in any of the observed patients during the follow up period of 18-64 months.

\section{Scoring of intraductal ultrasonographic findings}

Based on the results of multiple regression analysis, (1) the presence of sessile tumour (intraductal or outside of the bile duct), (2) tumour size greater than $10.0 \mathrm{~mm}$, and (3) interrupted wall structure were used as positive factors to predict malignancy on IDUS findings, and the relationship between intraductal ultrasonographic findings and final diagnosis was retrospectively analysed. As shown in table 5, in cases with no evidence of malignancy on histological findings of the biopsy specimen $(n=31)$, if IDUS findings showed two 
Table 5 Scoring of intraductal ultrasononographic findings and frequency of malignancy on final diagnosis

\begin{tabular}{lc}
\hline $\begin{array}{l}\text { No positive factors } \\
\text { on IDUS }\end{array}$ & $\begin{array}{l}\text { Frequency of malignancy on final } \\
\text { diagnosis* }\end{array}$ \\
\hline $\begin{array}{l}\text { (1) Total cases }(n=62) \\
0\end{array}$ & $2 / 17(12 \%)$ \\
1 & $5 / 7(71 \%)$ \\
$2-3$ & $37 / 38(97 \%)$ \\
\multicolumn{2}{l}{ (2) Cases with no evidence of malignancy on the histological findings } \\
of biopsy specimen (n=31) \\
0 & $0 / 15(0 \%)$ \\
1 & $2 / 4(50 \%)$ \\
$2-3$ & $11 / 12(92 \%)$ \\
\hline
\end{tabular}

Positive factors in predicting malignancy on intraductal ultrasonography (IDUS ) findings: (1) presence of sessile tumour (intraductal or outside of the bile duct); (2) tumour size greater than $10.0 \mathrm{~mm}$; and (3) interrupted wall structure.

*The process of final diagnosis is shown in table 4

or three positive factors, $92 \%$ of patients showed malignancy on the final diagnosis. In contrast, if IDUS showed no positive factor, no patient showed malignancy on the final diagnosis when the biopsy specimen showed no evidence of malignancy.

\section{Complications}

One patient suffered from acute pancreatitis which resolved within 48 hours with intravenous fluids and analgesia. No other complications occurred as a result of ERCP, IDUS, biopsy, or ENBD.

\section{DISCUSSION}

Accurate characterisation of biliary strictures is particularly important in patients with benign stenoses, as most of these patients can be successfully treated by the transient placement of biliary endoprostheses rather than surgery. ${ }^{27}{ }^{41}$ However, to our knowledge, there is no previous study which clarifies the management of patients in whom the endoscopic transpapillary biopsy specimen shows no evidence of malignancy. In this study, we utilised addition of IDUS for this purpose. Of course, improving methods of tissue sampling is a better strategy for enhancing reliability than requiring an additional imaging modality. Currently, the sensitivities of fluoroscopically guided transpapillary bile duct biopsy, brush cytology, and conventional bile cytology are $31-81 \%, 44-66 \%$, and $30-50 \%$, respectively. ${ }^{1-11}$ It is obvious that these results are unacceptable and that it is necessary to develop some way to compensate for these limitations. Bile duct biopsy under percutaneous transhepatic cholangioscopy (PTCS) is accurate and has a sensitivity of $93-96 \% .^{12} 27$ However, this requires an invasive procedure and is not routinely performed by endoscopists in Western countries.

Several studies have described the use of IDUS in patients with pancreatic ${ }^{15}$ or biliary strictures. ${ }^{32}$ In these studies, both benign and malignant strictures ultimately were resected. ${ }^{15} 32$ These studies provide valuable information about the accuracy of IDUS. However, the aims of the present study were not only to compensate for the false negative rate of ERCP obtained biopsies but also to prevent unnecessary surgical exploration. Previous reports have defined benign diseases as those with negative biliary duct samplings which remained cancer free during an observation period of six months, ${ }^{1}$ eight months, ${ }^{4} 14$ months, ${ }^{11} 15$ months, ${ }^{10} 18$ months, ${ }^{7}$ or 24 months. ${ }^{6}$ Therefore, we followed patients for at least 18 months to confirm that the pathology was in fact benign.

According to a preliminary study, PTCS guided biopsy of polypoid-type cholangiocarcinoma is more sensitive than biopsies of other types of cancer as cancer cells are present throughout the entire bile duct mucosa in this lesion. ${ }^{12}$ PTCS guided biopsy of metastatic tumour was less sensitive than biopsies of primary bile duct carcinoma. ${ }^{12}$ According to another previous study which compared percutaneous transhepatic IDUS findings and the findings of PTCS guided biopsy, tumour size greater than $10.0 \mathrm{~mm}$ and interrupted wall structure on IDUS images were factors that predicted malignancy. ${ }^{27}$ During the period of preliminary study in these reports, we predicted that the combination of transpapillary biliary biopsy and IDUS contributes to the management of patients with biliary strictures, and performed the current prospective study.

As IDUS shows asymmetric bile duct wall thickening due to inflammatory changes as well as cancer, ${ }^{17} 232627293234$ accurate characteristics of the demonstrated lesion are required. Menzel et al reported that when hypoechoic masses with irregular margins and inhomogeneous echo poor areas invading surrounding tissue on IDUS were considered malignant, the accuracy, sensitivity, and specificity of these IDUS findings were $89.1 \%, 91.1 \%$, and $80 \%$, respectively. ${ }^{32}$ If IDUS shows tumour invasion into the hepatic artery, the portal vein, or the pancreatic parenchyma, it suggests malignant disease, as previously reported. ${ }^{14} 15-222425313435$

In our study, endoscopic biopsy of the polypoid lesions showed $100 \%$ sensitivity in patients with cholangiocarcinoma. However, the biopsy was not sensitive in polypoid lesions with tumour thrombi caused by HCC. Thomsen et al reported a similar case of tumour thrombi caused by HCC but this patient's hepatic mass was not detected by ultrasonography prior to peroral cholangioscopy. ${ }^{42}$ Therefore, when biopsy of a polypoid lesion located at the hepatic hilus is negative, tumour thrombi caused by HCC should be considered. Although autoimmune deficiency syndrome related polypoid lesions of the bile duct have been reported in Western countries, we had no experience of this disease. ${ }^{43}$ On the other hand, the distal end of the bile duct is a common location of benign polypoid lesions including cholesterol polyps, adenomyomas, and inflammatory polyps. ${ }^{44-46}$

Our study also showed the limitation of biopsy in patients with the tumour outside of the bile duct, including pancreatic cancer. Kubota and colleagues ${ }^{1}$ and Sugiyama and colleagues ${ }^{4}$ also reported that the sensitivity of biopsy in pancreatic cancer was inferior to the sensitivity in primary bile duct cancer. Others recommend endoscopic ultrasonography guided aspiration cytology for these patients. ${ }^{47}$

Our current study indicated that (1) the presence of sessile tumour (intraductal or outside of the bile duct), (2) tumour size greater than $10.0 \mathrm{~mm}$, and (3) interrupted wall structure were positive factors in predicting malignancy on IDUS findings. As shown in table 5, in cases with no evidence of malignancy on histological findings of biopsy specimen, if IDUS findings show none of these three factor the presence of biliary malignancy is rare. In particular, patients with polypoid lesions located at the intrapancreatic duct and those with localised wall thickening who have some history (bile duct stones and others) may be conservatively observed. However, when IDUS shows two of these three factors, patients should be judged as having malignancy even if histological findings of biopsy specimen show no evidence of malignancy.

In our subjects, if patients with evidence of malignancy on histological findings of biopsy specimen or some positive factors on IDUS were judged to have malignant diseases, and the remaining patients were judged to have benign diseases, $95 \%$ $(59 / 62)$ were appropriately treated with three false positive and no false negative results.

In conclusion, if the endoscopic biopsy of a biliary stricture shows no malignancy, the patient may be managed using IDUS in combination with other diagnostic imaging and clinical findings.

.................

Authors' affiliations

K Tamada, T Tomiyama, S Wada, A Ohashi, Y Satoh, K Ido, K

Sugano, Department of Gastroenterology, Jichi Medical School,

Yakushiji, Tochigi 329-0498, Japan 


\section{REFERENCES}

1 Kubota Y, Takaoka M, Tani K, et al. Endoscopic transpapillary biopsy for diagnosis of patients with pancreaticobiliary ductal strictures. Am J Gastroenterol 1993;88:170-6.

2 Pugliese V, Conio M, Nicolo G, et al. Endoscopic retrograde forceps biopsy and brush cytology of biliary strictures: a prospective study. Gastrointest Endosc 1995:42:520-6.

3 Ponchon T, Gagnon P, Berger F, et al. Value of endobiliary bruch cytology and biopsies for the diagnosis of malignant bile duct stenosis: results of a prospective study. Gastrointest Endosc 1995;42:565-72.

4 Sugiyama M, Atomi Y, Wada N, et al. Endoscopic transpapillary bile duct biopsy without sphinctectomy for diagnosing biliary strictures. Am J Gastroenterol 1996:91:465-7.

5 Howell DA, Parsons WG, Jones MA, et al. Complete tissue sampling of biliary strictures at ERCP using a new device. Gastrointest EndosC 1996;43:498-502

6 Jailwala J, Fogel EL, Sherman S, et al. Triple-tissue sampling at ERCP in malignant biliary obstruction. Gastrointest Endosc 2000;51:383-90.

7 Ryan ME. Cytologic brushings of ductal lesions during ERCP. Gastrointest Endosc 1991;37:139-42

8 Kurzawinski TR, Deery A, Dooley JS, et al. A prospective study comparing brush and bile exfoliative cytology for diagnosing bile duct strictures. Gut 1992;33:1675-7.

9 Ferrari AP, Lichtenstein DR, Silvka A, et al. Brush cytology during ERCP for the diagnosis of biliary and pancreatic malignancies. Gastrointest Endosc 1994;40:140-5.

10 Mansfield JC, Griffin SM, Wadehra V, et al. A prospective evaluation of cytology from biliary strictures. Gut 1997;40:671-7.

11 Glasbrenner B, Ardan M, Boeck W, et al. Prospective evaluation of brush cytology of biliary strictures during endoscopic retrograde cholangiopancreatography. Endoscopy 1999;31:712-7.

12 Tamada K, Kurihara K, Tomiyama T, et al. How many biopsies should be performed during percutaneous transhepatic cholangioscopy to diagnose biliary tract cancer. Gastrointest Endosc 1999;50:653-8.

13 Engstrom CF, Wiechal KL. Endoluminal ultrasound of the bile ducts. Surg Endosc 1990;4:187-90.

14 Yasuda K, Mukai H, Nakajima M, et al. Clinical application of ultrasonic probe in the biliary and pancreatic duct. Endoscopy 1992;24:370-5.

15 Furukawa T, Naitoh Y, Tsukamoto $Y$, et al. New technique using intraductal ultrasonography for the diagnosis of diseases of the pancreatobiliary system. Ultrasound Med 1992;11:607-12.

16 Cushing GL, Fitzgerald PJ, Bommer WJ, et al. Intraluminal ultrasonography during ERCP with high-frequency ultrasound catheters. Gastrointest Endosc 1993;39:432-5

17 Gress F, Chen YK, Sherman S, et al. Experience with a catheter-based ultrasound probe in the bile duct and pancreas. Endoscopy 1995;27:178-84.

18 Tamada K, Ido K, Ueno N, et al. Preoperative staging of extrahepatic bile duct cancer with intraductal ultrasonography. Am J Gastroenterol 1995;90:239-46.

19 Tamada K, Ido K, Ueno N, et al. Assessment of portal vein invasion by bile duct cancer using intraductal ultrasonography. Endoscopy 1995;27:573-8.

20 Tamada K, Ido K, Ueno N, et al. Assessment of hepatic artery invasion by bile duct cancer using intraductal ultrasonography. Endoscopy 1995;27:579-83.

21 Tamada K, Ido K, Ueno N, et al. Assessment of the course and variations of the hepatic artery in bile duct cancer by intraductal ultrasonography. Gastrointest Endosc 1996;44:249-56.

22 Tamada K, Ido K, Ueno N, et al. Assessment of the pancreatic parenchymal invasion in bile duct cancer by intraductal ultrasonography. Endoscopy 1996;28:492-6

23 Tamada K, Kanai N, Ueno N, et al. Limitation of intraductal ultrasonography in differentiating between bile duct cancer in stage $T$ and stage T2: in-vitro and in-vivo studies. Endoscopy 1997;29:721-5.

24 Itoh A, Goto H, Naitoh Y, et al. Intraductal ultrasonography in diagnosing tumor extension of cancer of the papilla of Vater. Gastrointest Endosc 1997;45:251-60.
25 Kanemaki N, Nakazawa S, Inui K, et al. Three-dimensional intraductal ultrasonography: preliminary results of a new technique for the diagnosis of diseases of the pancreatobiliary system. Endoscopy 1997;29:726-31.

26 Tamada K, Tomiyama T, Ichiyama $M$, et al. Influence of biliary drainage catheter on bile duct wall thickness as measured by intraductal ultrasonography. Gastrointest Endosc 1998;47:28-33.

27 Tamada K, Ueno N, Tomiyama T, et al. Characterization of biliary strictures using intraductal ultrasonography: comparison with percutaneous cholangioscopic biopsy. Gastrointest Endosc 1998:47:341-9.

28 Tamada K, Yasuda Y, Tomiyama T, et al. Preoperative assessment of congenital bile duct dilatation using intraductal ultrasonography. Gastrointest Endosc 1999:49:488-92.

29 Tamada K, Tomiyama T, Oohashi A, et al. Bile duct wall thickness measured by intraductal US in patients who have not undergone previous biliary drainage. Gastrointest Endosc 1999;49:199-203.

30 Tamada K, Kanai N, Tomiyama T, et al. Prediction of the histologic type of bile duct cancer using intraductal US. Abdom Imaging 1999;24:484-90.

31 Tamada K, Tomiyama T, Ohashi A, et al. Preoperative assessment of extrahepatic bile duct carcinoma using three-dimensional intraductal US Gastrointest Endosc 1999;50:548-54.

32 Menzel J, Poremba C, Dietl KH, et al. Preoperative diagnosis of bile duct strictures-comparison of intraductal ultrasonography with conventional endosonography. Scand J Gastroenterol 2000;35:77-82.

33 Chak A, Isenberg G, Kobayashi K, et al. Prospective evaluation of an over-the-wire catheter US probe. Gastrointest Endosc 2000:51:202-5.

34 Tamada K, Wada S, Ohashi A, et al. Intraductal US in assessing the effects of radiation therapy and prediction of patency of metallic stents in extrahepatic bile duct carcinoma. Gastrointest Endosc 2000;51:405-1 1 .

35 Tamada K, Sugano K. Diagnosis and non-surgical treatment of bile duct carcinoma: developments in the past decade. J Gastroenterol 2000;35:319-25

36 Wada S, Tamada K, Tomiyama T, et al. Endoscopic microwave coagulation therapy for bile duct cancer with intraductal ultrasonographic monitoring: brief case report. Am J Gastroenterol 2000:95: 1 104-5.

37 Ohashi A, Ueno N, Tamada K, et al. Assessment of residual bile duct stones with use of intraductal US during endoscopic balloon sphincteroplasty: comparison with balloon cholangiography. Gastrointest Endosc 1999:49:328-3.

38 Ohashi A, Tamada K, Tomiyama T, et al. Influence of bile duct diameter on the therapeutic quality of endoscopic balloon sphincteroplasty. Endoscopy 1999;31:137-41.

39 Komatsu $\mathbf{Y}$, Kawabe T, Toda N, et al. Endoscopic papillary balloon dilation for the management of common bile duct stones: experience of 226 cases. Endoscopy 1998;30:12-17.

40 Tamada K, Higashizawa T, Tomiyama T, et al. New ropeway-type bile duct biopsy forceps with a side-slit for a guidewire. Gastrointest Endosc 2001;53:89-92.

41 Born P, Rosch T, Bruhl K, et al. Long-term results of endoscopic and percutaneous transhepatic treatment of benign biliary strictures. Endoscopy 1999;31:725-31.

42 Thomsen GH, Kruse A, Petersen A. Hepatocellular carcinoma presenting as a tumour of the hilar and extrahepatic bile ducts. Eur $J$ Gastroenterol Hepatol 1998;10:803-4.

43 Collins CD, Forbes A, Harcourt-Webster JN, et al. Radiological and pathological features of AIDS-related polypoid cholangitis. Clin Radiol 1993:48:307-10.

44 Ido K, Kawamoto C, Tamada K, et al. Diagnostic value of laparoscopic transcystic cholangioscopy. Endoscopy 1996;28:638.

45 Ojima H, Takenoshita S, Nagamachi Y. Adenomyoma of the common bile duct: report of a case. Hepatogastroenterology 2000;47:132-4.

46 Lauffer JM, Baer HU, Maurer CA, et al. Adenomyoma of the distal common bile duct mimicking cholangiocarcinoma. Dig Dis Sci 1998;43:1200-4

47 Erickson RA, Sayage-Rabie L, Beissner RS. Factors predicting the number of EUS-guided fine-needle passes for diagnosis of pancreatic malignancies. Gastrointest Endosc 2000;51:184-90. 